The eminent Professor of Midwifery in Edinburgh makes the following pertinent remarks. He says: "Formerly medical practitioners seem to have thought little, and medical writers said little, regarding the very repulsive and revolting character of the operation of craniotomy, when performed, as it frequently was, when the child was still living. Apparently, some obstetric practitioners and writers of the present day continue to look upon the practice of craniotomy as one that should not unfrequently be adopted, and one which it is quite justifiable to adopt. Obstetric reports and collections of cases have been published within the last few years describing craniotomy as performed forty or fifty times, or oftener, by the hand of the same practitioner. But, perhaps, ere long, it will become a question in professional ethics, whether a professional man is, under the name of a so-called operation, justified in deliberately destroying the life of a living human being.'

Woman naturally is mild, kind, and humane. She is endowed with great fortitude and undaunted courage. She has generally a great desire for offspring, and has a great love for children. 'Then how can we suppose that any woman with a well regulated mind, if fully aware of her responsibility, could willingly be a consenting party to the repeated destruction of her unborn infants? According to my own knowledge, the case is otherwise. I feel convinced in my own mind that there would scarcely be a woman to be found, who would not suffer any amount of bodily pain to save her infant.

Every woman in whom there exists organic impediment to the passage of a mature or full-grown infant, ought to be at proper time fully informed of the nature and as to the degree of the obstacle. She should also be made acquainted with the alternative operations which are suitable to meet her case. If the obstruction be moderate in degree, then the forceps, turning, or the induction of premature labour, will be proper ; but if these means are not available, or if the cause of difficulty is great in degree, then the performance of the Cæsarean section will be required.

The New Drsease! Telegrams received from Sir A. Buchanan, St. Petersburg, report that the fevers in that city are believed to have no affinity with the plague, but are attributed to over-crowded lodgings of the labouring classes, spoiled vegetables, and bad water. This is, indeed, only what most sensible persons had already concluded. The number of fever cases admitted into the hospitals varies from 100 to 150 a day, of which the average mortality has been 25 or 30 a day, and the highest mortality 60 . The Paris Moniteur of Saturday published a note, stating that the Government had made inquiry into the condition of St. Petersburg; and found that, although deplorable some weeks ago, it is now greatly improved, and shows no epidemic beyond the ordinary sickness of the season.

Tapeworm in Abxssinia. A recent traveller in Abyssinia, Mgr. Van den Deck, has sent to the Belgian Royal Society an interesting account of the tapeworm which so commonly aftlicts the inhabitants of that country. They take small doses of kousso only to get rid of the body, when the worm gets of an inconvenient size; but they are anxious to retain the head, for they are terrible gluttons, and having a tenia enables them to eat so much more. The tenia common in Abyssinia is T. mediocanellata.

\section{Gitiginal Commurnixations.}

\author{
RETROSPECTIVE NOTES ON OUT- \\ PATIEN'T PRAC'TICE.
}

By C. M. Durrant, M.D., Physician to the East Suffolk and Ipswich Hospital.

[Concluded from page 318.$]$

Miscellaneous Affections.

The list of those diseases that could be grouped according to their regional situation have been completed; it remains only to enumerate those affections which are of a miscellaneous character, and which cannot be included in the preceding classification.

1. Ancemia. 'The first on the list, as having afforded the largest number of cases, is anæmia. This is a blood-disease, and of very common occurrence among the out-patients of a hospital.

The chief causes of this affection among the poor are insufficient diet, too close confinement, and the existence of those diseases which drain the blood of its red corpuscles, as hæmorrhoids, menorrhagia, carcinoma, or any other exhausting discharge.

Among the middle and upper classes, another, and a very rife cause obtains, which is too much overlooked, and too often unheeded, both by practitioner and patient. I allude to the overworking of the brain, which is becoming daily in this age of competition an increasing cause of disease. An exhausted nervous system will as certainly produce an impoverished condition of the blood as insufficient food, or too powerful and prolonged muscular exertion; and cases arising from the former cause, will be found to be more resisting and more unsatisfactory to treat, than those originating in physical disturbance merely.

A few words on the condition of the heart, to which the attention of the patient is so mainly and so distressingly directed, may not be out of place. The irritable action of the organ itself, and the vascular murmurs peculiar to anæmia, are too well known to require comment ; but one point, I believe, is not sufficiently attended to, viz.: the importance of making such a thorough examination of the heart and vessels as will enable the practitioner to assure the patient and friends that no disease of the organ exists. I refer to this circumstance from having been so frequently told by anæmic persons that they have been informed that "their heart is not quite right," or " that the heart is slightly amiss." Now all this unnecessarily alarms the patient, aggravates the functional disturbance, and might be entirely prevented by a careful examination, and a faithful explanation of the precise cause of the disturbed action. The vascular murmurs which are sometimes temporarily heard in anæmic cases, both in the subclavian regions and also over the cartilage of the third rib on the left side, are quite distinct from the functional souffe and the bruit de diable, which is so common an attendant upon this condition of the blood.

Dr. Richardson, in his Asclepiad essay upon the former murmur, believes it to arise either from a peculiar condition of the subclavian muscle affecting the artery, or from pressure from behind the vessel caused by local tuberculous deposit. In highly anæmic subjects, I imagine that it may arise from a portion of condensed lung, not necessarily tuberculous, temporarily pressing upon a highly irritated artery, and causing a murmur by disturbing the current of blood through the vessel; or it may arise from 
spasm of the walls of the artery, unconnected with pressure.

In the treatment of anæmia, steel of course must be regarded as the chief remedy; but as the digestive organs are often much vitiated, due attention should be given in the first instance to their correction. In administering steel, it should be borne in mind that the more soluble the preparation, the more easily it will be assimilated. In the anæmia arising from an exhausted nervous system, the combination of steel with zinc, and dilute phosphoric acid with very small doses of strychnia, will be useful.

2. Cachexia. Closely allied to anæmia, and of which we are seldom without examples, is that peculiarly depraved condition of the blood recognised as cachexia. While anæmia is attributable to a diminution of red-globules, cachexia is traceable to a poisoned condition of blood, chiefly from without, the blood containing also effete matters, that it is unable to throw off. The exciting causes producing the cachectic condition, when not specific, as from syphilis, cancer, or tubercle, may generally be traced to unwholesome atmospheric influences, or to some of the long train of constitutional depressants which operate so surely upon the labouring poor.

Among the most constant of the exciting causes of this deteriorated condition of the general health, may be mentioned insufficient ventilation, and the inhalation of noxious blood-poisoning matters from imperfect drainage. These causes will, I fear, continue to operate until we can convince the working classes of the importance of fresh air by night, as well as during the day, and to which at present, they are most averse; and also to show them the dangerous results which follow prolonged exposure to the effluvia from human and animal drainage, to which they appear equally regardless and insensible.

In the treatment of cachexia, the removal, as far as possible, of these noxious causes of the malady just referred to, will be of the first moment. If the disease be fully established, an improved diet allowing meat and vegetables, with claret or cider, if they can be obtained, will be proper. Attention to the digestive organs, with the chlorate of potash, and afterwards the nitro-muriatic acid, followed by arsenic and cod-liver oil, will all tend to correct the depraved condition of the blood. Thorough cleanliness of the skin (a difficult matter to enforce among outpatients) must also be insisted on.

3. Rheumatism. Acute rheumatism, as a rule, is not an out-patient's disease. The forms which it has chiefly presented, have been chronic rheumatism, lumbaro, and chronic rheumatic arthritis. The majority of the cases of chronic rheumatism appeared to be quite independent of any previous attack of the acute form of the disease, and were not usually complicated by heart affection. Simple chronic rheumatism very frequently owes its origin to long continued derangement of the digestive organs, with vitiated secretions, producing blood-poisoning; it may arise also from prolonged exposure to wet and cold. One case was clearly attributable to gonorrhœa, and in two or three instances the muscular fibres as well as the tendons were implicated, constituting myalgia.

In the treatment of chronic rheumatism, strict attention should be paid to the digestive organs, for not only will their derangement produce the disorder in the first instance, but it will be impossible to cure it, so long as malassimilation to any extent exists. With this view, I have found nothing better at the onset than the bicarbonate and nitrate of potash with the tincture of colchicum, and spirit of nitrous ether. Although the beneficial influence of colchicum is doubted by some authors, I still think that I have often witnessed excellent results from its use in the 368 treatment of chronic rheumatism, as distinct from gout. In lumbago, it certainly is very useful. After. a time the iodide of potassium, with or withont the $\vec{F}$ addition of arsenic, may be advantageously added to $\stackrel{\mathcal{P}}{\square}$ the mixture. Cod-liver oil is also a very valuable 0 remedy in all stages of the disease, provided that the $\frac{}{\sigma}$ stomach be in a state to receive it. As an external $\overline{\bar{\omega}}$ application, cotton-wool covered with oiled silk, will $\vec{\nabla}$ generally give ease in the first instance; and after- $Q$ wards, a succession of small blisters or the iodine paint, applied daily to the affected part, will be of decided service.

In lumbago, a large plaster to the loins, composed of equal parts of opium and pitch plasters, will afford much comfort from its support. If myalgia complicate the attack, rest, a nutritious diet with stimu- $\delta$ lants, and cod-liver oil with steel, will be the proper 3 treatment.

Chronic rheumatic arthritis frequently commences i at the climacteric period in both sexes, and it $N$ is then a very obstinate disease. In the severer forms of this affection, whatever treatment may be $\sigma$ adopted, the result is too often doubtful; or if improvement obtain, it shews itself very slowly. As a 윽 tonic and improver of the blood, I believe that a $\vec{c}$ combination of arsenic and the iodide of potassium $\vec{G}$ with cod-liver oil, will be found the best. As local $D$ measures, warm douches with "shampooing," have 을. been strongly recommended. I think that I have seen benefit follow the application of the actual $\infty$ cautery, but it is a remedy that can seldom be had recourse to in practice. It was formerly much and advantageously employed in the Edinburgh Infirmary.

Under whatever form of rheumatism the patient suffer, he should be thoroughly clad in flannel, and, while taking a nutritious diet, he should avoid malt

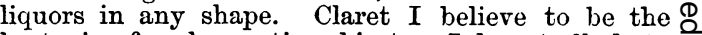
best wine for rheumatic subjects. I do not allude to the treatment by mineral baths and waters, as they can only be adopted by the wealthy.

4. Struma. Examples of almost every phase of $\supsetneq$ this frightfully common affection has been presented during the two years, and the worst cases that have obtained, have been among the out-patients living in the country. This circumstance may in part be attributable to the greater frequency of intermarriages among blood relations, which is a very common prac- $\frac{0}{3}$ tice among our agricultural poor; and in part also, to the insufficient and innutritious food to which the labourer and his family are often limited; and as a third cause, I would mention the crowded and illventilated bedrooms, and the consequently impure $\mathrm{O}$ air which, during the long hours of the night, they $N$ are doomed to breathe. In the treatment of struma of generally, the correction of the circumstances just $D$ alluded to, will be of the first consideration, for how many cases of phthisis can be traced back to a strumous dyspepsia, engendered by insufficient ventilation and improper food.

The poor themselves, as is well known, are unhap $\omega$ pily very averse to admitting air at night into their overcrowded bedrooms; and even those in a class above them, are too ready to exclude the amount of $\mathbb{D}$ air which is really necessary to a healthy assimila- $\stackrel{\infty}{-}$ tion. In reference to medicines, the first endeavour should be to correct the disordered condition of the primo vice, and for this purpose the alkalies with the chlorate of potash, and perhaps, the hypophosphites, will be the best remedies. Afterwards, as a tonic, no $\mathbb{\Phi}$ remedy will answer better than quinine, to which, if $\varrho$ necessary, may be added steel, and the iodide of potassium with cod-liver oil.

A difference of opinion still exists in reference to the management of strumous abscesses in the neck. 
Many are in favour of early lancing; my own preference leans to allowing them, as a rule, to break spontaneously under the influence of iodine paint or poultices. After the abscesses have broken and been sufficiently poulticed, the ointment of the iodide of lead will be found an excellent application.

Strumous enlargement of the tonsils is another very troublesome form of strumous constitutional disturbance. As a local application, I generally direct a solution of the nitrate of silver to be painted on the part once daily (a scruple to the ounce); but by many the tincture of iodine applied in the same manner is preferred.

Strumous otitis yields best to blistering behind the ear, correcting the digestive organs, and then giving cod-liver oil and quinine, with calomel and rhubarb twice a week as an alterative. I need scarcely add that the patient should be warmly clad in flannel. Anything indeed that can promote the formation of healthy blood, and prevent or improve depraved nutrition, must be sedulously recommended.

5. Bronchocele. Enlargement of the thyroid gland is an affection of very common occurrence among our out-patients ; and it has not departed from the general rule of having presented much more frequently in the female than in the male. As internal medicines, I have generally given large doses of the liquor potassæ with the chlorate of potash, in doses of from five to ten grains. As an outward application, the iodine paint or the compound iodine ointment have been the preparations used.

6. Purpura. We have had a few cases of the simpler form of this affection. Although defined by Willan as a skin-disease, purpura is essentially a blood-disorder, in which, under a peculiar condition of system, the blood-corpuscles become disintegrated and diffused. The cases referred to appeared to depend upon passive congestion of the liver in the first instance, which in its turn produced exhausted nervetone, and malnutrition, with the purpuric spots.

In the treatment, after regulating the diet, which should be light, but mixed and nutritious, the liver should be thoroughly emptied either by repeated doses of senna with sulphate of magnesia, or by castor-oil. After this, the mineral acids or the tincture of the sesquichloride of iron, with or without quinine, will be the best remedies. In the low form of chronic purpura, with slow, draining, internal hæmorrhage, the oil of turpentine or the ammonio-sulphate of iron will often act beneficially.

7. Homorrhoids. The examples of this affection, included as medical cases, have been simply external or internal piles, but not sufficiently chronic or severe to require surgical treatment. I have always regarded this often painful malady as one of defective nerve-tone, and depending chiefly upon congestion of the portal circulation. With this view, while giving every night at bedtime a scruple each of magnesia and precipitated sulphur, I have always prescribed with the best effect, at the same time, an alkaline mixture, with full doses of aromatic spirit of ammonia and chloric ether. Cod-liver oil is also a medicine of much value in hæmorrhoidal disease.

\section{Concluding Observations.}

I have now completed the running commentary upon the chief class of cases which have come under my notice as out-patients in the last two years. That medicine, as a science, is steadily advancing is unquestioned, and its chief progress is founded upon an increasing knowledge of minute physiology and pathology.

Disease is now not recognised as it was formerly, as being a something per se, a distinct and separate entity, and differing altogether from the normal condition; but it is now regarded simply as a perversion of the natural and physiological phenomena, which are uninterruptedly taking place during health, but which, under the influence of various external and internal exciting causes, so affect the normal vital processes, as to constitute malnutrition or disease. The subject of perverted nutrition is largely occupying the attention of physiological and pathological inquirers, and the investigation of the influence which nerve-nutrition or its opposite exert upon the causation of disease, is full of practical interest and importance.

It is now generally admitted, that the tendency of most diseases, when unaided by treatment, is towards recovery, and that the influence of drugs is more correctly shewn in guiding to a termination in health, rather than by attempting abruptly to cut short the progress of the malady.

In order, however, to reduce our knowledge of the effects of medicines upon disease to as much exactitude as possible, it is, as I remarked at the commencement of these papers, very important that the results of individual remedies be faithfully and carefully collected and reported. For this purpose, nothing but " combined medical observation" can avail, and in no way can this be better effected than by inducing the individual members of our large Association to give a brief record of the results of their own treatment, and to test and report on the treatment of others.

Whatever course be adopted for the obtaining this result, whether by a reissue of schedules, or by any other method of inquiry, I would merely suggest the importance of condensing and simplifying the questions as much as possible, so that answers from the fully engaged practitioner may not be wanting.

\section{NOTES ON HERNIA.}

\section{By John Thompson, M.D., F.R.C.S., Bideford. [Continued from p.221.]}

WHEN reduction of a strangulated hernia by the taxis and its aids is found impracticable, nothing remains but the operation by incision. It happens but too frequently that the patient either objects to this, or requests delay and further consultation. The difficulty of managing him may thus be more trying than the treatment of his disease. Keeping this in mind, it is generally wise at the outset to say that, in case the efforts with the hand, etc., do not succeed, it will be necessary to use the knife. A timid person often becomes reconciled to an operation when time is allowed him to make up his mind; and the more early this is done, the better the prospects of the case.

In provincial practice the results of operations are so generally known, and so long remembered, especially when they are unfavourable, that it is matter of importance, for the welfare of future sufferers, that an established operation should not suffer in reputation, which it must do, if performed only as a last resort, when pain and agony compel compliance. It is most notable how strong is the logic of facts on the mind of a sufferer; let him know that others afflicted as he is, have successfully passed through the operation, and your point is almost gained; but, on the other hand, sinister information of unsuccessful cases equally disposes in the opposite direction. Feeling that next in importance to the performance of the operation is the question of time, I have dwelt the more on these matters, which, though simple, may be of considerable consequence. 